\section{Preliminary Results on the Regulatory Role of IFN-g and IL-10 Human Schistosomiasis Mansoni}

\author{
Silvia ML Montenegro/ ${ }^{+}$, \\ Paulo Miranda*, Frederico GC Abath, \\ Kirte M Teixeira, Eridan M Coutinho, \\ Ana LC Domingues*, \\ Luis Domingues*, Joeli Brinkman, \\ Itay Gonçalves, Siddhartha \\ Mahanty**, Alan Sher***, \\ Thomas A Wynn***
}

Departamento de Imunologia, Centro de Pesquisas Aggeu Magalhães-Fiocruz, Av. Moraes Rego s/no, Cidade Universitária, 50670-420 Recife, PE, Brasil

*Laboratório de Imunopatologia Keizo Asami

LIKA-UFPE, Recife, PE, Brasil **Centers for

Disease Control, Atlanta, GA, USA

***National Institutes of Health, Bethesda, MD, USA

Key words: Schistosoma mansoni - spleen cells IL-10 - IFN-g

Studies about in vivo IL-10 immunoregulation demonstrated that $\mathrm{CD}^{+}{ }^{+}$cells from Schistosoma mansoni infected mice produced large amounts of IL-10 and that this response was temporally and mechanistically linked to the downregulation of the Th1 cytokine response (A Sher et al. $1991 \mathrm{~J}$ Immunol 147: 2713-2716). The addition of neutralizing anti IL-10 mAb to the cultures resulted in an increase in IFN-gproduction to levels approaching those seen before the egg laying phase (EJ Pearce \& A Sher 1991 Exper Parasitol 73: 110-

This work was supported by World Health Organization, Special Programme for Research and Training in Tropical Diseases and Fundação de Amparo à Ciência e Tecnologia.

${ }^{+}$Corresponding author. Fax:+55-81-453.1911.E-mail: silvia@cpqam.fiocruz.br

Received 4 May 1998

Accepted 31 August 1998
116). Thus, it is clear from in vitro studies on the regulation of cytokine production and the observation of the Th1 and Th2 profiles during $S$. mansoni infection in mice that Th1 and Th2 responses are counter-regulatory. However, the role of IL-10 in controlling the cytokine response may be dependent on the immunological background of the host. T Wynn et al. (1997 J Immunol 159: 5014-5023) studied S. mansoni egg induced granuloma formation and cytokine production in knockout mice which suggested that IL-10 may play a key role in controlling the development of both type 1 and type 2 responses.

The present study aimed to compare the cytokine pattern in acute and chronic schistosomiasis patients and to study the contribution of IL10 and IFN-gto the regulation of Th1 and Th2 responses.

The patients selected for the study presented acute $(n=7)$, intestinal $(n=6)$, hepatointestinal $(n=1)$ or hepatosplenic $(\mathrm{n}=9)$ schistosomiasis mansoni as defined previously (AD Coutinho \& ALC Domingues 1987 Mem Inst Oswaldo Cruz 82: 335337). The control group was composed of healthy Brazilian individuals.

The cytokine pattern elicited in response to soluble egg antigens (SEA) and soluble worm adult preparation (SWAP) were compared in acute and chronic patients. In the absence of IL-10 neutralization, the acute patients responded to SEA and SWAP by producing significant quantities of IFN-g when compared with the chronic patients. There was also a significant IL-10 response in cells obtained from acute and chronic patients. Neutralization of IL-10 had no significant effect on the already high levels of IFN-g produced in response to SEA in acute patients, while IFN-g production was significantly high in chronic patient cells, suggesting that the cytokine regulatory mechanisms may be different in acute and chronic infection. Neutralization of IFN-gresulted in similarly increased levels of IL-10 in acute and chronic patients, while IL-4 and IL-5 were not affected, suggesting a counter-regulatory role between IFN-g and IL-10. When spleen of hepatosplenic patients were used in the experiments, the results were similar to those found in peripheral blood mononuclear cells, suggesting that there is no anatomic differences in the expression of cytokines in antigen stimulated cultures where IL-10 was neutralized. These studies demonstrate that early infection with $S$. mansoni is associated with a significant IFN-gresponse and IL-10 plays an important down-regulatory role in that response during late infection. 
174 IFN-g and IL-10 in Human Schistosomiasis - Silvia ML Montenegro et al. 